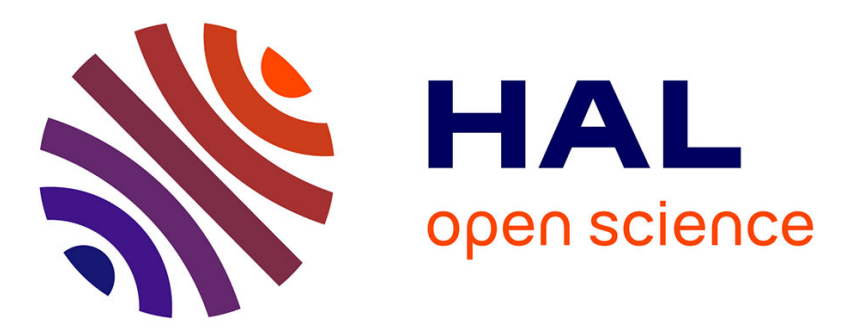

\title{
Catégorisations professionnelles des demandes masculines de chirurgie esthétique et transformations politiques de la médecine
}

\author{
Yannick Le Hénaff
}

\section{- To cite this version:}

Yannick Le Hénaff. Catégorisations professionnelles des demandes masculines de chirurgie esthétique et transformations politiques de la médecine. Sciences Sociales et Santé, 2013, 31 (3), pp.39 - 64. 10.3917/sss.313.0039 . hal-01684124

\section{HAL Id: hal-01684124 https://hal.science/hal-01684124}

Submitted on 3 Mar 2018

HAL is a multi-disciplinary open access archive for the deposit and dissemination of scientific research documents, whether they are published or not. The documents may come from teaching and research institutions in France or abroad, or from public or private research centers.
L'archive ouverte pluridisciplinaire HAL, est destinée au dépôt et à la diffusion de documents scientifiques de niveau recherche, publiés ou non, émanant des établissements d'enseignement et de recherche français ou étrangers, des laboratoires publics ou privés. 


\section{CATÉGORISATIONS PROFESSIONNELLES DES DEMANDES MASCULINES DE CHIRURGIE ESTHÉTIQUE ET TRANSFORMATIONS POLITIQUES DE LA MÉDECINE} Yannick Le Hénaff

John Libbey Eurotext | « Sciences sociales et santé »

2013/3 Vol. 31 | pages 39 à 64

ISSN 0294-0337

Article disponible en ligne à l'adresse :

https://www.cairn.info/revue-sciences-sociales-et-sante-2013-3-page-39.htm

\section{Pour citer cet article :}

Yannick Le Hénaff, « Catégorisations professionnelles des demandes masculines de chirurgie esthétique et transformations politiques de la médecine », Sciences sociales et santé 2013/3 (Vol. 31), p. 39-64.

DOI $10.3917 /$ sss.313.0039

Distribution électronique Cairn.info pour John Libbey Eurotext.

(C) John Libbey Eurotext. Tous droits réservés pour tous pays.

La reproduction ou représentation de cet article, notamment par photocopie, n'est autorisée que dans les limites des conditions générales d'utilisation du site ou, le cas échéant, des conditions générales de la licence souscrite par votre établissement. Toute autre reproduction ou représentation, en tout ou partie, sous quelque forme et de quelque manière que ce soit, est interdite sauf accord préalable et écrit de l'éditeur, en dehors des cas prévus par la législation en vigueur en France. Il est précisé que son stockage dans une base de données est également interdit. 
Sciences Sociales et Santé, Vol. 31, $n^{\circ} 3$, septembre 2013

\title{
Catégorisations professionnelles des demandes masculines de chirurgie esthétique et transformations politiques de la médecine
}

\author{
Yannick Le Hénaff*
}

Résumé. Cet article analyse le travail de catégorisation effectué par les chirurgiens plastique sur les clients hommes demandeurs d'opérations esthétiques. Sur la base de 80 entretiens avec des praticiens, nous mettons en évidence une appréciation largement genrée de leur clientèle. Les hommes sont ainsi considérés comme des clients à part, voire relégués au prétexte de l'impossibilité de modifier «naturellement » leur corps ou de pathologie latente. Nous mettons alors en lien les transformations politiques observables dans le monde médical - et en particulier l'évolution des modèles de relation médecin-client - et l'hétérogénéité des discours de ces praticiens sur la demande de chirurgie esthétique.

Mots-clés : chirurgie esthétique, catégorisation sociale, genre, transformations politiques.

\footnotetext{
* Yannick Le Hénaff, sociologue, Université de Rouen, Laboratoire DYSOLA, rue Lavoisier, 76821 Mont Saint-Aignan Cedex, France ; yannick.le-henaff@univ-rouen.fr

Mes remerciements vont aux référés de la revue et à Stéphane Héas pour leurs remarques stimulantes.
} 
«Le bon chirurgien, c'est simple, il se reconnaît à sa clientèle ». Cette formulation leitmotiv dans le monde de la chirurgie esthétique (1) invite à questionner les caractéristiques d'une «bonne » clientèle et, par extension, les caractéristiques du «bon » praticien. Les discours des professionnels, souvent qualifiés de rhétoriques, sont en sociologie largement appréhendés comme des manifestes visant à imposer un point de vue sur l'activité (Damien et Tripier, 1994), circonscrivant l'espace de la pratique mais aussi de son public. Désigner le client («bon » ou « mauvais ») engage plus avant à influer (et plus exactement à tenter d'influer) sur ses attentes légitimes et sur les résultats qu'il est en droit d'espérer, ainsi que sur les normes interactionnelles devant régir cette relation (Hughes, 1996). Rendre compte de ces catégories - telles qu'elles sont édictées par les professionnels - permet donc en retour de caractériser le vrai travail.

Ces catégorisations sont ici questionnées à partir d'une population numériquement marginale, les hommes, représentant $10 \%$ de la clientèle (Knipper et Jauffret, 2003) dans une profession majoritairement masculine à $81 \%$ (Le Breton-Lerouvillois, 2007). Cette focale emprunte aux positions de Hughes (1996) selon lequel les catégories s'accordant le plus difficilement au cadre de l'ordinaire sont également les plus à même de receler un intérêt sociologique : par leur moindre résistance à l'analyse, elles découvriraient plus aisément ce qui ailleurs reste dissimulé. Notre posture traite en partie d'une intervention elle-même marginalisée : la pénoplastie, opération visant l'agrandissement du pénis, non remboursée par l'Assurance maladie.

En analysant ces catégorisations sociales comme des révélateurs de la culture professionnelle, nous tentons d'en dessiner les contours, en cla-

(1) La chirurgie esthétique est définie par l'Assurance maladie comme le pôle non pris en charge de la chirurgie plastique reconstructrice et esthétique. Elle se focalise essentiellement sur quelques interventions portant sur les seins (augmentation ou diminution), les amas graisseux (liposuccion), les paupières (blépharoplastie), le ventre (plastie abdominale), le visage (lifting) ou le nez (rhinoplastie). Ces catégorisations ne sont toutefois ni étanches, ni consensuelles. Certaines interventions sont aux frontières de ces deux champs : la chirurgie de réduction mammaire est, par exemple, prise en charge par l'Assurance maladie dès lors que 300 grammes de glandes sont retirés. Cette distinction est également travaillée par les clients, mais aussi par les chirurgiens qui, en négociant en particulier l'aspect thérapeutique de certains de leurs gestes en chirurgie esthétique, tendent à les définir à partir d'un besoin et non d'un désir (Jacobson, 2000). 
rifiant la définition du « bon » client (2), mais également en soulignant les contrastes entre praticiens, dans une approche segmentée des mondes professionnels (Strauss, 1992). Les praticiens pratiquant l'esthétique ne constituent en effet pas un groupe homogène aux frontières clairement définies. S'ils sont majoritairement chirurgiens plastique, d'autres spécialités sont autorisées à intervenir " esthétiquement » sur la zone anatomique dont ils ont la charge : les chirurgiens en maxillo-facial, ophtalmologie, otorhinolaryngologie et stomatologie peuvent ainsi intervenir sur la face, les chirurgiens gynécologues sur les seins et les chirurgiens urologues sur le sexe de l'homme. Tous les chirurgiens plastique ne développent pas, à ce titre, une activité esthétique. Certains, plutôt des hospitaliers, se concentrent exclusivement sur la chirurgie plastique reconstructrice quand d'autres se focalisent sur la chirurgie esthétique. Entre ces deux positions, la majorité de ces praticiens optent pour une activité mixte.

Nous nous intéressons particulièrement aux répercussions des transformations politiques de l'espace médical émettant l'hypothèse qu'elles favorisent l'émergence de discours hétérogènes. Ces discours recueillis en entretien sont analysés comme des constructions ou des «figures idéologiques » (Broussard et al., 2006 : 224) imprégnées des valeurs du groupe. A ce titre, l'espace médical ne peut être considéré comme en dehors des influences sociales et culturelles ; son langage est d'ailleurs un site privilégié de l'expression des différentes déclinaisons du genre (Fox Keller, 1992). La capacité de ces catégories à dire l'axiologique se double ici d'un engagement prescriptif, ayant prise sur la réalité professionnelle (Austin, 1970). Pour autant, si ses effets doivent être considérés comme performatifs, ils ne présagent pas systématiquement des pratiques (Weller, 1994).

Notre démarche sociologique s'appuie principalement sur un corpus d'entretiens réalisés auprès de 80 chirurgiens plastique français entre 2007 et 2010 lors d'une recherche doctorale. Un échantillonnage, au sens strict

(2) En faisant référence au « client » et non au «patient», il ne s'agit surtout pas d'affirmer que le client est «le demandeur et le juge de toute production et de toute activité » (Broussard et al., 2006 : 210), mais de développer une posture en rupture avec les rhétoriques des professions « prestigieuses ». Le terme de patient est pétri de jugements de valeurs et constitue une description idéalisée de l'individu en attente de réponse médicale, mais aussi de la relation avec le médecin. En analysant l'organisation de l'activité médicale - et non seulement la chirurgie esthétique - comme n'importe quelle autre relation de service, avec ses spécificités, mettant aux prises un professionnel et un client dans un environnement concurrentiel, on s'autorise les comparaisons irrévérencieuses (Hughes, 1996). 
du terme, n'a pas été effectué, mais ces chirurgiens ont été sollicités selon un principe de diversification maximale entre les différents statuts, le sexe et la région d'installation. Ils se rapprochent des données démographiques recensées par l'Ordre des médecins (Le Breton-Lerouvillois, 2007), sans pour autant s'y conformer totalement. Âgé de 51 ans (contre 48 ans pour la recension nationale), l'enquêté type est un homme (à $85 \%$ contre $81 \%$ ), libéral (à $85 \%$ ), exerçant principalement en Île-de-France (31\%), mais aussi en Provence-Alpes-Côte d'Azur (19\%), Rhône-Alpes (20 \%), et Pays-de-Loire (9,5\%). Enfin, et en accord avec les analyses de Jaisson (2002), ces chirurgiens apparaissent largement comme des " héritiers mâles ». Leurs origines sociales découvrent une forte prégnance des professions médicales et des cadres supérieurs pour le père (près de troisquarts des enquêtés sont regroupés sous ces deux catégories), alors que la mère est largement sans profession (dans près de 2 cas sur 3 ), enseignante ou de profession paramédicale (près d'un tiers pour ces deux catégories).

Des observations non participantes lors de congrès professionnels ou durant leur activité ont également été réalisées sur une durée de trois semaines. Notre présence a toutefois été limitée lors des consultations effectuées dans un cadre privé, en raison des réticences exprimées par les praticiens libéraux, ce qui apparaît comme un problème classique auquel est confronté le sociologue de la santé (3). Une analyse de contenu des revues professionnelles a été faite, et l'une d'entre elles, les Annales de Chirurgie Plastique Esthétique, a fait l'objet d'un traitement systématique. Dans un deuxième temps, des plateformes Internet de discussion portant sur la pénoplastie ont été investiguées par le biais d'une analyse de contenu. Ces fora de discussion sont des espaces d'échange dans lesquels sont partagés questionnements, angoisses, expériences ou savoirs (Aubé et Thoër, 2010). Chacun de ces matériaux a été analysé comme un discours particulier, nécessitant d'être réinscrit dans le contexte de son énonciation et, en particulier, en fonction des conditions de production et du public visé (Kaufmann, 1996)

La première partie de l'argumentaire éclaire les distinctions induites entre le client et la cliente. Elles mettent en évidence le caractère socialement et médicalement construit de l'intérêt des femmes pour leur apparence, ce qui les imposerait comme les clientes « naturelles » de la chirurgie esthétique, contrairement aux hommes dont la demande génère le malaise. Nous spécifions alors les modèles de justifications utilisés par ces chirurgiens afin de reléguer les demandes masculines, ou au moins de

(3) Voir aussi Guyard (2002) pour le cas des consultations des gynécologues-obstétriciens. 
les suspecter. Enfin, ces catégorisations sont considérées au regard des transformations politiques qui agitent la médecine depuis trente ans et, en particulier, de la place accordée au client et à ses desiderata dans le processus de décision.

\section{Une appréciation sexuée de la demande esthétique}

Dans cette partie, les éléments caractérisant la femme comme un «bon » client seront présentés. Ils seront associés à la communauté de valeurs viriles que constitue le monde de la chirurgie.

\section{Une cliente légitime}

Dans la littérature professionnelle, comme dans les discours des enquêtés, la clientèle est essentiellement présentée comme féminine, admettant chez les femmes une attention supposée plus « naturelle » pour leur apparence. Dans une formulation restée célèbre dans ce monde professionnel, le chirurgien Vilain affirmait ainsi que "le matin, devant le miroir, l'homme se rase, la femme s'inquiète. Il se jauge, elle se juge » (Villain, 1987 : 267). L'homme pourrait négliger, dans une certaine mesure, son apparence corporelle ou feindre de le faire. Cette polarisation incline, pour certains de ces praticiens au moins, à distinguer l'approche diagnostique et technique de ces clientèles, comme le préconise Lévignac: "Notre abord ne sera pas le même pour l'homme et pour la femme. Pour l'homme, nous croyons qu'il est beaucoup plus important d' "avoir de la gueule" que d'être beau. En fait, c'est "avoir de la gueule" qui est être beau! (...) Nous agirons avant tout pour libérer sa personnalité. Pour la femme, notre propre réponse est ici celle d'un homme. On cherchera surtout à dégager plus de grâce et de féminité »(Lévignac, $1988: 78)$.

Dans cette acception s'affirme une nette tendance à l'individualisation de l'esthétique chez l'homme où les expériences qui jonchent son passé doivent s'inscrire sur le corps. Précautionneusement d'ailleurs, le terme de beauté est banni ou presque du vocabulaire («avoir de la gueule » versus «être beau»). Ces discours, particulièrement prégnants jusque dans les années 1980, deviennent beaucoup moins audibles par la suite, dans la revue savante au moins. Certes, le féminin reste de rigueur pour évoquer la clientèle, mais l'inflexion n'en est pas moins palpable. Un souci de neutralité incline à la recherche d'un discours à valeur scienti- 
fique dans une discipline où l'institutionnalisation et la légitimité sont longtemps restées incertaines. Ces précautions rhétoriques ne présagent toutefois pas de l'attitude des praticiens qui, au contraire, prolongent ces discours dans l'entre-soi particulièrement, mais aussi parfois face au sociologue, surtout quand il est homme (4). L'adhésion à ces propos, ni totale, ni consensuelle, laisse toutefois entrevoir une communauté de valeurs qui joue le rôle de terreau dans lequel puiser et s'imprégner, sans qu'elle ne prédéfinisse discours et positions.

\section{Une communauté de valeurs viriles}

Ces discours nécessitent d'être appréhendés dans leur environnement social, celui du monde de la médecine et plus encore de la chirurgie, intensément marqué par le genre. Dans le tableau de la masculinité dressé par Jaisson (2002), la chirurgie domine avec $87 \%$ d'hommes. Bercot et Mathieu-Fritz (2006), mais aussi Zolesio (2012), livrent quelques éléments de la faible attractivité de ces disciplines pour les étudiantes en médecine : cooptation défavorable, forte implication professionnelle limitant les possibilités d'articulation à la sphère privée et représentations largement sexuées. L'imaginaire de ce groupe professionnel associe les qualités socialement construites comme masculines aux exigences supposées de cette activité, comme l'autorité, la responsabilité, l'acuité décisionnelle ou bien encore la combativité (Cassell, 1998 ; Katz, 1999).

La violence symbolique de certains propos, même dans cet univers masculinisé, interpelle. À trois reprises au moins lors de cette enquête, des théorisations paralogiques très ouvertement misogynes, et revendiquées comme telles, ont été tenues en entretien par des chirurgiens hommes, appelant à un universalisme culturel et historique de cette inégalité des sexes.

(4) En confrontant mes observations avec celles de R. Ghigi (2007), ayant effectué un terrain d'enquête auprès de chirurgiens plastique italiens, quelques spécificités de l'entretien en tant que rapport social de sexe ont pu être notées. Le jeu de séduction, parfois le regard médical et l'évaluation de ces spécialistes sur le corps de l'enquêteur s'inscrivaient de façon plus ostentatoire face à la sociologue alors que l'homophilie de genre a pu autoriser certains praticiens hommes à tenir des propos empreints de violence symbolique à l'égard des femmes. 


\section{Julien T. (5) et sa théorie de la «baisabilité »}

Julien T. : "La chirurgie esthétique ne consiste pas à rendre les gens beaux ou jeunes, non. C'est juste un moyen. Le but, c'est de rendre les gens, si vous me permettez le terme, "baisables" (...) Elles n'ont pas de sein ou elles sont moches, ou elles ont des grosses fesses, et elles se disent : "si je reste comme ça, je ne vais jamais trouver le prince charmant". Moi j'interprète ça : il faut que je les rende baisables. Il faut qu'elles trouvent le prince charmant qui descende de son cheval pour la prendre. Avec tous les sens du mot prendre. Vous êtes un homme, je peux vous dire ça (...) Et c'est pas forcément celles qui en ont besoin qui viennent nous voir. C'est pas forcément les moches. C'est celles qui ont un doute sur leur capacité à séduire. Pourquoi celles qui sont déjà séduisantes veulent encore plus? Et ça c'est particulièrement sensible avec les seins, l'augmentation. Et bien parce qu'elles sentent bien que si elles avaient plus, si elles étaient plus séduisantes, elles pourraient taper des hommes mieux. Plus haut encore (...).

Enquêteur : Et pourquoi les hommes hétéros ne viennent pas plus vous voir?

Julien T. : Parce que les hommes hétéros ne sont pas des baisables, ce sont des baiseurs. Tout est là-dedans. Quel est son problème? Gagner du fric pour avoir du pouvoir. Avec ça, il aura un fort potentiel de baisabilité active. Pas passive. Voilà. Un homme hétéro ne cherche pas à être séduisant (...) Je suis un grand misogyne. Je prends les femmes pour ce qu'elles sont.»

Replacées dans le contexte de l'interaction, face à un enquêteur inconnu, ces affirmations vont à l'encontre de la retenue supposée des individus composant les fractions élevées de la hiérarchie sociale (Bourdieu, 1982). L'exposé de Julien T. tranche également avec les discours des autres professionnels, ce qu'il confirme d'une certaine manière en reconnaissant sa misogynie. Il semble rompre sciemment cette convention, ce qui est peu arrivé dans le cadre de ce terrain. Pourtant, cet écart révèle vraisemblablement les logiques professionnelles à l'œuvre, ce verbatim faisant écho à des propos entendus dans les coulisses de ce monde, en marge des colloques ou lors d'observations en salle d'opération, soit

(5) Afin de conserver l'anonymat de ces enquêtés, les prénoms ont été modifiés. 
dans les rares moments où, dans le cadre de cette enquête, le sociologue se trouve au cœur du groupe professionnel. Par ailleurs, la banalité supposée de ces propos témoigne d'une communauté de valeurs masculines où ce n'est pas seulement le chirurgien qui est défini par son genre, mais plus largement sa culture professionnelle et la structure des relations entre professionnels et clients, mais aussi entre professionnels et sociologue.

\section{La clientèle masculine, un malaise latent}

Si elles sont distinguées des motifs des femmes, les intentions des hommes sont souvent appréhendées avec circonspection, en particulier chez les praticiens davantage que chez les praticiennes qui s'expriment de manière plus nuancée. Le contact en consultation s'établirait plus facilement, plus «naturellement », voire même plus «saine(ment) » avec une femme, soulevant a contrario le spectre pathologique chez l'homme. De telles déclarations sont parfois avancées sans pouvoir être étayées par des données concrètes, et encore moins épidémiologiques, relevant ainsi de l'évidence incorporée. En outre, ces demandes sont fréquemment associées à une « communauté » gay, supposant un rapprochement établi $a$ priori entre homosexualité et souci de l'apparence (6), la consultation ne s'engageant que rarement, de leur propre aveu, sur les orientations sexuelles de leurs clients. Cette association prend davantage de poids quand, en creux, des comportements de femmes leur sont agrégés, en opposition à des attendus masculins. En deçà de l'évidence socialement construite, cette association présuppose la déviance.

«C'est plus simple pour moi que ce soit des femmes en consultation. Parce que la démarche esthétique me paraît claire. Pas chez un mec. Un mec qui fait autant attention à son esthétique quand il est jeune, ça m'inquiète. Je trouve qu'un mec, c'est machiste, ça devrait avoir une certaine rusticité quelque part. Même si on fait attention à soi. Le mec, il se regarde à 20 ans, au lieu de regarder les femmes » (Erwan R., libéral quinquagénaire)

L'inquiétude qui anime Erwan R., tout en reproduisant les fondements hétéronormatifs du groupe - il est « normal » de « regarder les femmes »- engage, en particulier quand ces propos sont portés par un

(6) Cet énoncé fait écho aux travaux médicaux et psychiatriques de la fin du XIX ${ }^{\mathrm{e}}$ siècle, qui ont largement contribué à la construction des stéréotypes sur l'homosexualité. Ceux-ci, tentant de désigner scientifiquement les indices révélant l'homosexualité, ont régulièrement dénoncé l'attention pour l'apparence physique (Tamagne, 2002). 
médecin, à soupçonner la maladie («ça m'inquiète »). Plus avant, cette suspicion trace les frontières entre soi et l'autre : la présence du pathologique induit, anthropologiquement, un rapport à l'altérité (Fainzang, 1999). Cet exercice cognitif corrobore une classification binaire hommefemme et homosexuel-hétérosexuel, inscrivant une demande dérangeante dans le cadre d'une a-normalité maîtrisée et socialement construite. En ce sens, ces catégorisations offrent des ressources cognitives qui durcissent, en situation délicate, les frontières de la «normalité » (Tamagne, 2002). Cette classification ne reste toutefois qu'une ressource et ne présage pas directement du traitement de la demande dont de nombreux aspects sont co-construits lors de la consultation. Ces propos correspondent à des images simplifiées de la réalité, ou basées sur quelques exemples ayant profondément marqué le chirurgien. C'est le cas de Xavier B. (libéral quinquagénaire), très réticent aux demandes masculines dont il soupçonne une base psychopathologique. Interrogé sur cette posture, il focalise sa méfiance sur l'action en justice intentée par l'un de ses anciens clients, selon lui injustifiée et révélatrice d'une «névrose».

Toutes les interventions ne sont d'ailleurs pas positionnées sur le même plan ; il existe une cartographie symbolique du corps (Le Breton, 1992) investissant les zones anatomiques selon leur importance dans la construction de la virilité : la chirurgie du sexe soulève particulièrement la crainte, et dans une moindre mesure la rhinoplastie, alors que d'autres actes sont considérés comme moins engageants, comme la liposuccion.

\section{Les justifications médicales et esthétiques de la relégation et du refus}

Le malaise qu'instaurent, par moments et chez certains professionnels, ces demandes masculines, engage ces praticiens à développer des techniques discursives de disqualification, voire de relégation, tout en faisant allégeance aux normes sociales de tolérance (Kaufmann, 1996). Cette logique argumentaire les déconsidère tout en évitant de se référer à une condamnation explicitement morale. Deux voies sont alors privilégiées, empruntant tour à tour ou parallèlement à la rhétorique du naturel et/ou à la pathologisation. 


\section{Des techniques limitées: l'impossible construction du naturel chez le client}

Dans ce monde professionnel, le « naturel », en tant que reproduction morphologique censément « normale » et «idéale », est considéré comme un objectif opératoire absolu. Cette qualité traverse tous les discours autour de cette pratique au point de faire l'objet d'une intense négociation. Elle s'est instituée en tant que référence dans laquelle chacun (industriel, praticien, client) puise pour se conforter et dénoncer l'autre. Jacobson (2000) relève la construction sociale sous-jacente à cette négociation sous forme d'oxymore (travailler la nature pour la rendre plus naturelle), et qu'elle nomme «paradoxe du naturel ».

Pour Astrid R. (praticienne-hospitalière trentenaire), les techniques chirurgicales à sa disposition ne sont pas suffisamment efficientes pour lui permettre de construire un nez réellement « masculin », ce qui la conduit à refuser systématiquement les rhinoplasties chez l'homme alors qu'elle les plébiscite chez la femme. Des procédés similaires consacreraient ainsi le « naturel » chez cette dernière quand ils condamneraient toute tentative chez l'homme. Ce «nez en trompette », «trop fin » qu'Astrid R. décrit comme le résultat opératoire inévitable s'oppose à la virilité, en tant qu'essence et expression de l'homme. L'idée qu'il doit posséder un nez «fort» (dixit) polarise les oppositions symboliques, assurant une similitude quasi physiognomonique entre le visage, les traits de caractère et le sexe. Ludovic D. (quadragénaire libéral) ne propose ainsi ces interventions que dans le cadre d'une masculinité strictement prédéfinie : "Ça m'est arrivé (de refuser) des hommes qui voulaient un petit nez fin, et je leur ai dit: "écoutez, pour moi c'est très clair, je ne fais pas de petit nez américain, vous êtes un homme, vous avez un visage longiligne, il vous faut un nez suffisamment long, il vous faut un nez qui reste fort, je ne souhaite pas vous faire ce type de nez". "

Des réticences plus moralisatrices se devinent ainsi sous l'argument butoir du naturel. Dans les précautions qu'il exprime à effectuer des rhinoplasties chez l'homme, Hagège qualifie tout autant le résultat que la démarche de « ridicule », postulant que l'individu portera irrémédiablement un stigmate indélébile, et cela même si les suites opératoires sont esthétiquement satisfaisantes : "Le petit changement très accepté par une femme sera souvent insuffisant chez un homme (...) et surtout quelque chose d'indéfinissable qui faisait dire à Sénéchal qu'une résection, même modérée, pouvait donner facilement à un homme "un nez ridicule et sans expression" ; et qui a fait dire à Pollet, un jour, en réponse à la demande d'un de ses amis, médecin de 50 ans, qui désirait une rhinoplastie : "tu 
auras peut-être un beau nez, mais tu auras certainement l'air ridicule” » (Hagège, 1988 : 297).

Malgré le «beau nez », la condamnation tombe, irrémédiablement : « tu auras certainement l'air ridicule ». D'autres praticiens mettent en avant leur incapacité à définir le beau chez l'homme. L'exaltation du naturel se mue en argument commode au refus ou à la délégation, et obscurcit la production d'une apparence normée. Cette exaltation démontre, après d'autres, comment la médecine participe à la construction même de la réalité de la nature, naturalisant ici une différence de sexe (Oudshoorn, 1994).

\section{Une demande pathologique}

Cette demande qui indispose glisse aisément dans le versant pathologique, autorisant un refus médicalement argumenté. Cédric L. (libéral quinquagénaire) soupçonne ainsi et a priori toutes les demandes masculines sur la base d'une instabilité psychologique : "Encore une fois, la demande esthétique est révélatrice (chez les hommes) de psychoses profondes dans un nombre de cas non négligeable. Alors qu'on n'a pas ça chez la femme. Les femmes, elles sont chiantes, elles sont pénibles, elles sont superficielles. Elles sont méchantes, elles sont tout ce que vous voulez. Mais elles ne consultent pas, ou rarement avec une psychose avérée. »

Les difficultés rencontrées sont bien distinguées selon l'appartenance sexuelle. Inhérentes à leur nature chez les femmes, elles signent chez les hommes un dysfonctionnement psychique, inéluctable ou presque. Quel que soit le résultat de l'opération, celui-ci est condamné par son insatisfaction consubstantielle dès lors qu'il est en demande de transformation esthétique. Il est même appréhendé par certains comme une menace, pouvant mener à la plainte. Si la dysmorphophobie - la crainte obsessionnelle d'un désordre dans la perception de l'image corporelle - est un trouble connu de ces praticiens, sa reconstruction professionnelle comme objet de mise en garde ou possibilité de refus apparaît sexuellement discriminée et largement surévaluée pour les hommes (Pitts-Taylor, 2007). Traditionnellement pourtant, le sens commun et médical attribue une prévalence des pathologies liées à la psyché aux femmes. Et cela, depuis le XIX ${ }^{\mathrm{e}}$ siècle, soit le moment où elles sont considérées comme essentiellement différentes et deviennent dès lors un objet de recherche privilégié (Lacqueur, 1992). En construisant alors l'homme comme la norme, tout ce qui s'en éloigne devient étrange : la femme, mais aussi celui qui, comme ici, ne se comporte pas selon certains critères de masculinité. Ce n'est donc pas seulement «le stéréotype de la "nature féminine" (qui) continue à contaminer les appro- 
ches les plus scientifiques » (Clément et Membrado, 2001 : 71), mais plus largement les stéréotypes de genre.

Dans le panel d'opérations disponibles, la pénoplastie concentre les soupçons, exacerbe les craintes et constitue pour beaucoup un acte à éviter. Elle est analysée, ici, non comme un révélateur du traitement des demandes masculines, mais davantage comme un éclairage des mécanismes de stigmatisation et d'évitement de certaines demandes. La posture $\mathrm{du}$ groupe professionnel est, en ce sens, symptomatique, mettant en garde contre l'acte («la prudence est toujours de mise avant une opération de transformation du pénis » est-il préconisé sur le site Internet chirurgiensplasticiens.info) et insistant sur l'approfondissement des motivations. Nombre de chirurgiens s'accordent sur le caractère particulier de cette demande en raison d'une prétendue implication psychologique, voire psychopathologique. Pour beaucoup, les motifs purement esthétiques sont remis en cause au profit d'une interprétation essentiellement basée sur une défaillance sexuelle refoulée. Dans une lecture para-psychanalytique très répandue chez ces praticiens, les "difficultés sexuelles » sont transférées chez ces clients sur un problème de «taille ». "C'est une population pénible (...), très particulière (...) L'homme qui veut augmenter la taille de son sexe, ou le diamètre de son sexe... c'est peut-être avant tout dans la tête » précise Maelle P. (libérale quadragénaire). Cette demande engage irrémédiablement la virilité défectueuse du demandeur. Erwan R. dénonce des problèmes d'érection, de "puissance » précise-t-il dans un vocabulaire évocateur. Cette impuissance sexuelle, corollaire d'un manque de confiance en soi, questionne l' "Homme » dans ses dispositions reproductrices, et au-delà même. Sexualité, réussite professionnelle, confiance en soi et hétérosexualité constituent ainsi les principaux aspects de la masculinité déficiente chez ces clients, associés différemment selon les praticiens, mais aussi selon l'opération. Pour la pénoplastie, c'est essentiellement la sexualité défaillante et la confiance en soi qui sont incriminées, et dans une moindre mesure la réussite professionnelle, quand la rhinoplastie n'engage que ces deux derniers aspects ; pour les autres opérations de chirurgie esthétique chez l'homme, la figure de l'homosexuel apparaît principalement.

Ces argumentaires font parfois état d'une controverse « scientifique » concernant la fiabilité des résultats obtenus, ce qui permet de refuser a priori cette intervention, en se réservant un droit de choix exclusif sur la «balance bénéfice/risque », pour reprendre la terminologie de ces professionnels, contestant au client toute possibilité d'estimation ou de hiérarchisation concurrente des priorités. La dimension sanitaire prime ici au détriment de la qualité de vie (Sarradon-Eck et Pelligrini, 2012). Or, cette balance constitue une thèse manipulable, renvoyant à la nature socia- 
lement construite du résultat espéré mais aussi à sa légitimité. Découlant a priori d'une logique économique, ce processus décisionnel recouvre des dimensions très variables, en particulier en chirurgie esthétique où le bénéfice se fond difficilement dans une nomenclature santé-maladie. D'ailleurs, elle recouvre des réalités diverses chez les chirurgiens, pour qui il peut s'agir de confort, de mieux-être psychologique, de la disparition d'un complexe ou bien encore du bouleversement de la vie du client transformé corporellement et socialement. Cette balance questionne surtout la place du client dans la construction de la décision. Longtemps, le modèle paternaliste a fait figure de référence, consacrant la toute puissance médicale. Mais ce modèle est aujourd'hui largement remis en cause, y compris au sein des professions médicales dans lesquelles se distinguent des postures, que nous nous attacherons désormais à restituer. Car si la réticence est palpable, les réponses de ces chirurgiens restent contrastées - tous ne refusent pas ces clients, ni tout le temps.

\section{Les transformations politiques et les postures médicales}

La catégorisation des demandes masculines, et des demandes de pénoplastie en particulier, n'est pas homogène dans ce groupe professionnel. Ces contrastes peuvent être reliés à l'évolution des formes politiques de la santé (Barbot, 2008) et de la configuration des rôles des différents acteurs. Après avoir rappelé le cadre de ces transformations, nous rendons compte des différentes postures, puis proposons quelques éléments concernant les clients en demande de pénoplastie.

\section{Des transformations politiques à l'œuvre}

Le monde médical français connaît, depuis les années 1980, un profond bouleversement dans l'économie et la légitimité des pouvoirs en place (Dodier, 2003). Les dispositifs législatifs et administratifs, les attentes de la population, mais aussi le renforcement des associations d'usagers engagent une redéfinition sensible de la relation médecin-client et une réévaluation du rôle de ce dernier, avec un accès favorisé à l'information. Pourtant, loin d'homogénéiser les pratiques médicales, cette évolution semble au contraire favoriser l'expression de leur diversité (Barbot, 2008). Au-delà de cette hétérogénéité, l'analyse souligne les tensions intra-professionnelles, mais aussi les vecteurs de ces contrastes ; en d'autres termes, elle précise comment et chez qui ces positions se sont construites. 
Le degré d'autonomie du client ainsi que la manière de penser l'objectivité des décisions médicales constituent les deux principaux opérateurs permettant de dégager deux formes politiques : la tradition clinique paternaliste, dont l'impact s'essouffle, et la modernité thérapeutique participative qui s'établit en contrepoids (Barbot, 2008). Ces deux modèles agissent comme des pôles auxquels se réfèrent les médecins. Dans le premier modèle, le médecin s'impose comme une figure scientifique, unique dépositaire de la décision et dont les prérogatives sont consolidées par la vulnérabilité supposée du client. La relation, mais aussi la légitimité du praticien sont fondées sur la confiance accordée, induisant la délégation du pouvoir sous le postulat de la totale intégrité morale et de l'engagement dévoué au service du bien-être de la communauté. Cette délégation de la décision du client vers le médecin ne porte pas uniquement sur le domaine clinique, mais s'étend également à des dimensions morales (7). Le refus de certains chirurgiens plastique d'intervenir sur les hommes, strictement ou pour quelques opérations, relève de cette logique dans laquelle, in fine, l'intérêt du client est toujours revendiqué, même si cela conduit à agir contre sa volonté.

Le second modèle, la modernité thérapeutique participative, s'est imposé progressivement ces trente dernières années, notamment par le biais d'aménagements juridiques et administratifs (8). Elle témoigne d'un changement de paradigme à l'égard du client qui est désormais engagé à participer activement aux processus décisionnels thérapeutiques le concernant. Si la modernité thérapeutique participative tend à s'imposer, elle n'a pourtant pas complètement absorbé ni effacé la tradition clinique paternaliste. Ces deux modèles se côtoient, s'entrecroisent, générant une pluralité de postures et de justifications, découvrant la diversité des conceptions de l'exercice médical.

(7) Barbot (2008) montre comment ce modèle a un temps légitimé la transfusion sanguine « à leur insu » des témoins de Jéhovah qui récusaient cette technique. Désormais, ces médecins sont dans une posture plus problématique par la collision de ces deux formes politiques, tiraillés entre le respect des conventions religieuses (induisant une place importante du client dans la décision le concernant) et l'absolue nécessité de mettre tout en œuvre pour préserver la vie.

(8) La loi du 4 mars 2002 portant sur le droit des malades confirme ainsi cette tendance, à laquelle viennent s'ajouter différentes directives, chartes, codes et autres recommandations : loi Huriet en 1988, loi de bioéthique en 1994, nouveau code de déontologie médicale en 1995, charte du patient hospitalisé rénovée en 1995, recommandations de l'ANAES en mars 2000, etc. 
Pour les questionner ici, notre principal analyseur est la pénoplastie, opération certes peu pratiquée, mais très présente dans les discours de ces praticiens, qui se situent régulièrement à son égard. La signification et l'investissement de cette position priment ici sur la réalité statistique. Toutefois, les postures relevées pourront pour certaines d'entre elles dépasser le cadre de cette demande - en particulier pour les deux premières.

\section{Des postures et des catégorisations contrastées}

Les postures identifiées correspondent à des idéaux types distingués à partir de discours aux caractéristiques exemplaires ; elles dessinent des tendances générales, auxquelles peuvent être associées des éléments de carrière particuliers.

\section{Le refus inconditionnel}

Pour les tenants du refus inconditionnel, l'intervention dépréciée est systématiquement refusée ou déléguée. Il peut s'agir d'une opération en particulier, la pénoplastie le plus souvent, mais aussi, plus rarement, de toutes les demandes masculines. Ces chirurgiens, qui refusent en bloc ces sollicitations d'hommes, disposent généralement d'une clientèle conséquente dont ils se félicitent. La saturation du planning opératoire offre la possibilité - certains d'entre eux parlent même de luxe - de sélectionner clientèle et interventions. Cette conjoncture se présente essentiellement chez les praticiens les plus âgés. Cette posture s'observe également chez les hospitaliers disposant d'une consultation privée, et dont une partie de l'activité est déjà satisfaite par leurs fonctions. La proximité aux sociétés savantes et syndicats module également les écarts à l'idéal professionnel. Les libéraux défendant cette position sont souvent proches et bien insérés dans ce tissu : ils briguent ou ont occupé des positions clés en leur sein, et s'investissent dans ces manifestations, colloques ou groupes de travail. La pression du groupe s'exerce intensément sur la pratique de ceux qui convoitent sa reconnaissance. L'âge, la saturation de la clientèle et la proximité des réseaux professionnels constituent trois des principales caractéristiques de cette position, et cela bien qu'elles ne soient pas des conditions sine qua none. Les chirurgiens refusant la seule pénoplastie découvrent ainsi un profil plus contrasté, et dans lequel se fondent de nombreuses chirurgiennes. Chez elles d'ailleurs, les autres demandes masculines sont appréhendées avec moins de réticences que leurs confrè- 
res (9). Ces praticiens sont imprégnés de l'idée que l'on construit sa clientèle dans le soin apporté à la sélection des «bonnes » indications, d'un point de vue physiologique et anatomique, mais aussi «psychologique » et social, ce qui d'une certaine manière les conforte dans leur positionnement. Avoir «la clientèle qu'on mérite » ou «à l'image du chirurgien » constitue l'un des poncifs de ce groupe.

Jonathan S. (sexagénaire libéral) est prototypique de cette posture : au-delà de la pénoplastie, il récuse systématiquement les demandes masculines, quelles qu'elles soient. Bénéficiant d'une solide réputation auprès de ses confrères, il s'est un temps investi au sein de la société savante en y occupant des postes prestigieux. A posteriori, il s'enthousiasme de la carrière qu'il a pu mener, et définit sa propre clientèle comme «belle ». Il est d'ailleurs considéré par ses confrères comme l'un des chirurgiens les plus en vue de la ville de province dans laquelle il consulte. Face aux demandes masculines, et donc aux pénoplasties, Jonathan S. présente donc des refus inconditionnels, explicitant et appliquant formellement ses catégorisations. Pour lui, il s'agit d'écarter l'homme, « mauvais » client par nature. Mais si cette demande l'incommode profondément, il lui est pour autant difficile d'expliciter aussi évidemment sa position, surtout en consultation. L'expression de ce refus n'est donc jamais formulée face au requérant ; il opte pour une délégation en amont, via son assistante chargée des prises de rendez-vous. Ce stratagème lui évite d'avoir à motiver les raisons de son refus et à effectuer un délicat travail de persuasion. Il n'est ainsi jamais en contact avec ces demandeurs.

\section{L'accord contraint}

Cet accord concerne les chirurgiens disposant d'a priori négatifs à l'égard de certaines interventions (la pénoplastie en premier lieu) ou de certains publics (les hommes), mais qui s'y adonnent toutefois. Accéder à la demande ne peut alors que générer le malaise.

Plutôt d'âge moyen, dont la réputation et la clientèle ne sont pas tout à fait établies, ces praticiens restent difficilement identifiables à partir d'indicateurs stables, peu de spécificités se dégageant réellement de leur carrière. C'est d'abord leur position qui les distinguent, à l'instar de Cédric L. Tout au long de l'entretien, il apparaît particulièrement désillusionné à l'égard des évolutions de sa pratique, tant vis-à-vis de «la paperasse qui nous submerge » que dans sa relation au client. Celui-ci serait

(9) Toutefois, seules 7 praticiennes (sur 12 enquêtées) se sont positionnées vis-à-vis de cette opération, ce qui ne permet pas de généraliser cette tendance. 
devenu plus exigeant et moins reconnaissant. Sa réticence à opérer les hommes, mais aussi à effectuer des pénoplasties, qu'il dépasse pourtant, l'engage à prendre davantage de précautions, en sondant notamment leurs potentielles velléités judiciaires : selon lui, ces clients seraient plus procéduriers et développeraient davantage d'indications psychopathogènes. Face à ce public, il s'applique donc longuement à mettre en garde, effectuant un important travail sur les motivations ; en insistant particulièrement sur les risques inhérents à toute opération chirurgicale, il invite à une réflexion approfondie. Ce travail lui permet à la fois de s'assurer la confiance du client mais aussi d'identifier (au moins de tenter d'identifier) des problèmes potentiels. Dans ce cas, il s'autorise «quelques fois » à refuser ceux qu'il estime trop menaçants, trop pointilleux ou revendicateurs.

Jonathan S. comme Cédric L. développent des catégorisations assez similaires, mais optent pour des réponses radicalement différentes. La dissonance chez ce dernier entre ses catégorisations et ses décisions est difficilement vécue et source d'inquiétude. Cette contrainte, générant la désagréable impression de ne pas tout maîtriser, écorne son identité professionnelle. Au contraire, Jonathan S., agissant en accord avec son travail de catégorisation, est conforté dans sa position puisqu'il n'est jamais confronté au client déconsidéré. Cette délégation lui sert d'enclave identitaire, préservant l'image qu'il projette de lui-même et de son activité. Il est à noter que cette posture ne concerne aucune chirurgienne enquêtée.

\section{L'accord a priori}

Une position plus libérale se détache dans laquelle l'homme n'est $a$ priori pas envisagé comme un «mauvais » client, bien qu'il ne puisse être tout à fait considéré à l'égal de la femme. Ces spécialistes, plutôt plus jeunes que la moyenne des chirurgiens plastique, accordent au client une latitude importante dans la décision finale. Les rôles et les tâches sont ainsi distribués : le praticien, technicien compétent, assure une information claire et exhaustive, quand le client, en consommateur éclairé, effectue ses choix. L'accord est de principe, mais engage parfois un travail de questionnement des motivations en consultation. Une seule enquêtée revendique cette position, qu'elle prend d'ailleurs soin d'associer à son internat durant lequel elle a traité de nombreuses pathologies de la verge.

Dimitri B. (quadragénaire libéral) ambitionne une pratique qu'il définit comme «moderne », mais que d'autres praticiens qualifieraient de «marchande ». Il n'hésite d'ailleurs pas à faire état de son appétence financière et de sa volonté d'accroître sa clientèle esthétique dans les années à venir au détriment de la chirurgie plastique reconstructrice. Il 
s'agit d'une volonté commune aux praticiens qui développent une vision concurrentielle de l'activité. Leur palette d'interventions est en général importante, intégrant notamment la médecine esthétique, traditionnellement moins pratiquée par les plasticiens et essentiellement constituée d'injections sous-cutanées, et ils usent fréquemment de sites Internet pour augmenter leur visibilité.

La pénoplastie peut être effectuée si le motif est clairement identifié comme physique. "Je n'en ai pas, ou peu (de demandes), mais je suis pas contre (la chirurgie du sexe de l'homme). Moi j'assimile un peu ça à la demande de chirurgie d'augmentation mammaire de la femme qui fait du $90 \mathrm{C}$ et qui veut des plus gros seins. J'ai une bite normale, et je la veux plus grosse. C'est une modification du schéma corporel qui au départ n'est pas anormal. Le mec il me dit : "j'arrive pas à bien baiser ma femme”, je lui dis : "c'est pas moi qui vais vous aider. Allez voir un psy. Si sexuellement vous avez pas de problème, bon, je vais changer votre image corporelle". Comme une femme qui veut des plus gros seins. C'est pour moi la même démarche, à partir du moment où vous avez décodé qu'il n'y a pas de problème sexuel.»

Dans le cadre de la modernité thérapeutique participative, le refus est plus difficilement recevable. Pour autant, l'accord à opérer ne lisse pas les conceptions morales, mais tend idéalement à les écarter du processus décisionnel. Disculpé de promouvoir cette transformation, le plasticien n'est alors qu'un agent répondant à une demande. D'ailleurs, et malgré leur acceptation, ces chirurgiens font explicitement référence au pathologique comme limite de leur geste, puisque tout «problème » est soigneusement évité. Ils font ainsi allégeance aux normes du groupe en n'écartant pas l'existence de défaillances sexuelles comme origine de cette demande. Proche de la posture précédente dans le processus décisionnel - on accepte d'opérer en veillant à écarter les cas définis comme problématiques - le travail identitaire diffère ici. Le sentiment de conserver la décision finale permet au praticien de se sentir au centre de son activité.

\section{L'accord militant}

D'autres enfin, comme Rémi S. (libéral quinquagénaire), affichent plus ostensiblement ces techniques en disgrâce, jusqu'à en faire des piliers de leur activité. Cette opération, délaissée par ses confrères, constitue une niche dans laquelle il a su se construire une réputation. Certains de ses clients font ainsi plusieurs centaines de kilomètres pour bénéficier de cette technique, et son nom - comme nous avons pu le vérifier - circule sur les forums Internet spécialisés. La principale caractéristique de ces praticiens, outre celles déjà mentionnées pour l'accord a priori, est leur relatif 
isolement vis-à-vis de l'épicentre du groupe professionnel. Le parfum de souffre qui émane de cette intervention les éloigne en effet de l'idéal véhiculé (10). Cette indépendance leur permet de tenir en haute estime un travail dévalorisé par leurs confrères. Rémi S. cultive ainsi d'autres réseaux, multipliant par exemple les voyages de formation à l'étranger. Il œuvre d'ailleurs essentiellement en chirurgie « ambulatoire », ce qui lui permet de libérer sa clientèle quelques heures seulement après l'opération, et surtout de travailler dans sa propre structure, évitant de faire appel aux installations d'une clinique privée.

Pour lui, l'acceptation de la pénoplastie est moins conditionnelle. L'intervention, jugée anodine, est d'abord perçue sous l'angle de la plusvalue qu'elle peut apporter à l'opéré et non, comme dans les deux premières postures et dans une moindre mesure dans la troisième, à partir de la pathologie potentielle. L'accord militant est essentiellement porté par des chirurgiens qui, à l'instar de Rémi S., effectuent régulièrement ce geste, au point d'en être banalisé. Il est à ce titre vraisemblable que l'importance prise par cette intervention dans leur carrière les a progressivement amenés à réévaluer leur position, et à revaloriser cet acte. Avoir été confronté à ce genre d'opérations et de clients durant sa formation apparaît en outre déterminant pour la proposer par la suite. Mais ils prennent toutefois soin de la justifier en se réinscrivant là aussi dans les valeurs de masculinité prônées par le groupe, et, en premier lieu, en soulignant qu'ils partageaient initialement les craintes de celui-ci quant aux aspects psychopathologiques de cette demande. Ils revendiquent donc être d'abord passés par la deuxième ou la troisième posture puis, après avoir multiplié cette intervention, avoir progressivement changé de regard sur cette clientèle. "Je me suis aperçu que ces opérations, finalement, il n'y avait pas de risque chirurgical, mais même psychologiquement, c'est pas du tout impliquant (...) Et la plupart du temps c'est le bon père de famille, le gars normal qui se fait ça, qui a une demande assez précise qui est de l'ordre du réaliste. Et puis souvent c'est des gens qui viennent en couple. »

Pour légitimer cette intervention, Rémi S. s'empresse de préciser, en accumulant les preuves, la « normalité » de cette demande, dont la figure paternelle est l'apogée. Le «bon père de famille » écarte de facto l' « anormalité », mais également, en symbole de l'individu établi, le soupçon de la recherche de performance sexuelle. La multiplication des conquêtes ou l'accroissement du plaisir de ces partenaires est systématiquement

(10) En affirmant cela, nous ne prétendons pas qu'il existe un idéal accepté de tous, mais, par simplification, qu'à l'intérieur des segments composant ce groupe, celui ayant prise sur cette société tend à développer un modèle particulier. 
dénoncé et considéré comme des motifs frauduleux par tous ces chirurgiens. La demande doit au contraire relever d'un complexe, s'inscrivant dans le cadre d'un axe thérapeutique et non pas devenir une technologie dopante. La perspective d'un " couple » uni écarte définitivement cet écueil. Cette demande viserait en fait à faire face au «syndrome de la douche collective » (dixit), en référence à l'imaginaire viril des hommes entre eux après l'effort, un lieu propre à l'attention intime de l'autre, mais également à l'humour potache. En identifiant cette demande dans une problématique visuelle, Rémi S. revirilise l'acteur qui la porte, excluant les problèmes de confiance en soi («c'est le gars qui sait où il va»). Plus avant, il précisera que sa clientèle, "à son image », est principalement composée d' "entrepreneurs », répudiant par là-même la figure de l'homme impuissant. Si aucune chirurgienne enquêtée n'est positionnée dans cette catégorie, lors de notre terrain de recherche au moins une femme était spécialisée dans cette pratique.

Que ce soit pour réfuter certaines pratiques ou au contraire les revendiquer, les registres discursifs puisent dans un creuset de valeurs similaires, sollicitées diversement voire inversement, travaillées de manière différente par les «formes politiques de la médecine 》(Barbot, 2008 : 1012) mais aussi par l'exercice au quotidien et la confrontation à certaines catégories de population. Dans le cas des pénoplasties, ceux qui s'y refusent dénoncent des problèmes d'impuissance et de transfert des difficultés sexuelles, alors qu'on affirme, chez ceux qui la pratiquent, éviter soigneusement ces demandes douteuses. Pour tous donc, la recherche d'une amélioration sexuelle et la pathologie psychique s'érigent comme les dangers principaux : elles définissent les limites au-delà desquelles l'acte ne peut être défini comme authentiquement médical (Strauss, 1992). Notons enfin qu'aucune différence de genre entre chirurgien et chirurgienne ne peut être énoncée sans risque de généralisation hâtive. Et cela, même si ces femmes apparaissent moins fermées aux demandes masculines, excepté pour la pénoplastie qu'elles sont très nombreuses à éviter.

\section{Du point de vue des clients}

Les postures de ces professionnels peuvent être considérées dans un cadre plus large, incluant - entre autres acteurs - les clients. Les fora de discussion Internet sur la pénoplastie constituent pour cela un terrain de recherche intéressant, en particulier dans le cadre d'une opération taboue, et pour laquelle il est difficile de solliciter des entretiens. À ce titre, leur investissement dans ces espaces est bien moins conséquent que celui des 
internautes naviguant sur les autres fora de chirurgie esthétique (11). Témoins de cette participation limitée, leurs avatars et leur fiche de renseignements sont rarement notifiés, et leur présence se borne aux moments de la recherche d'informations, parfois à la narration de leur expérience. Les possibilités de mobilisation collective sont en ce sens limitées, tout comme les revendications face au dédain de cet acte dans le monde médical.

Le décalage entre le discours de ces professionnels et celui de ces internautes interpelle. Ces sites ne rendent pas ou très peu compte des réticences professionnelles. Cette dissociation est liée aux dispositifs existant autour de la chirurgie esthétique pour choisir son opérateur et ainsi éviter d'errer de praticiens en praticiens : en plus des plateformes de discussion, les sites personnels des chirurgiens ou de leur clinique fournissent nombre d'indications (12), parmi lesquelles les interventions effectuées. Sur les fora, les noms d'une demi douzaine de chirurgiens reviennent d'ailleurs très régulièrement, nommément ou par leurs initiales. Il est ainsi aisé de savoir qui solliciter, dans un contexte où l'usage d'internet à des fins d'information de santé croît (Méadel et Akrich, 2010). À l'image des mobilisations associatives s'opposant à des points de vue médicaux (Loriol, 2003), ces sites fournissent une liste des «bons » médecins, soit ceux qui accomplissent cet acte. Pour beaucoup, cette prise de renseignements s'inscrit dans un parcours sur le long terme, à l'instar de Jérémy P. (trentenaire) pour lequel s'écoulent, entre ses premières recherches et son intervention, près de deux ans et demi. "J'ai tout d'abord commencé par me renseigner sur les différentes solutions (...) Une fois le type d'intervention choisi, j'ai regardé sur le net, dans des forums, des avis de chirurgiens, des cliniques tout ce qui concernait la chirurgie par injection de graisse extraite par notre propre organisme (...) Les sites que j'ai visités ont été nombreux (...) Il me restait à trouver une clinique, toujours sur Internet.»

Internet constitue un moyen de contournement des vecteurs d'information traditionnels (médecins ou proches) qui, dans le cas de la pénoplastie, peuvent s'avérer inopérants, l'intervention étant peu connue des

(11) Ces utilisateurs sont principalement des femmes, facteur pouvant expliquer cette différence, d'abord car elles sollicitent davantage Internet pour obtenir des informations de santé, mais aussi, ce qui lui est directement lié, car ce sont elles qui principalement prennent en charge cette question au sein de la famille et de l'entourage (Cresson, 1998).

(12) Contrairement aux autres spécialités, la chirurgie plastique (et surtout la chirurgie esthétique) a largement investi cet espace. Des sites comme cosmedia.fr ou esthéticon.fr se sont d'ailleurs spécialisés dans la comparaison des prix et des pratiques. 
généralistes, et parfois mal perçue. "J'ai franchi le pas pour la première fois en décembre 2004, je l'aurai bien fait avant mais je ne savais pas où m'adresser. J'avais été voir un médecin généraliste (...) et un urologue aussi. Les deux ont été incapables de m'aider » (Bernard, trentenaire). Les interventions culturellement teintées trouvent ici un espace de visibilité. Ces dispositifs pallient, à la fois, l'absence de système d'information publique et l'opacité régnant autour d'un marché où la dynamique concurrentielle est limitée (Karpik, 1995). Ces recherches permettent en outre au client de multiplier les points de vue sur son problème, et de produire une décision « armée ». Ces modes de communication constituent donc bien des leviers pour celui-ci, lui permettant d'être davantage acteur de sa santé.

\section{Conclusion}

Derrière ce qui s'annonce comme un dogme, l'intérêt des femmes pour leur apparence et la suspicion qu'engendre une demande masculine, s'élève l'horizon de la construction sociale du corps et ici du sens et du traitement accordés à une demande de modification. Doit-elle être considérée comme un acte luttant contre une pathologie ou un geste de confort? Entre-t-elle dans le cadre des missions de la médecine ? Ici, les prétentions éthiques dont se couvre la profession médicale pour plébisciter ou refuser telle intervention ou telle clientèle laissent apparaître les influences des stéréotypes de genre. L'étiquetage pathologique, par exemple, par le biais du diagnostic de trouble mental, n'apparaît donc pas seulement lié à un sexe, féminin, mais à une déviance : la femme par rapport à la référence que constitue l'homme (Lacqueur, 1992) ou l'homme en demande de modification corporelle qui s'écarte d'une manière d'être attendu. Si, comme le soulignent Clément et Membrado (2001), les médecins ont contribué à développer l'idée d'une nature féminine, celle-ci doit être perçue dans son rapport à une nature masculine, qui est également esquissée chez ces professionnels, et non isolément.

Malgré l'apparente homogénéité de ce groupe professionnel, les positions et catégorisations de ces praticiens se contrastent vis-à-vis de cette demande masculine et des pénoplasties en particulier. Ces contrastes relativisent l'approche homogénéisée de ces chirurgiens esthétiques dans la littérature, exclusivement ou presque perçue sous le prisme d'une domination masculine radicale (Bordo, 1989 ; Morgan, 1998). Ces perspectives conduisent, assez paradoxalement, à polariser les rôles attendus de ces praticiens, voire à les naturaliser selon leur sexe. Le travail de catégorisation doit d'ailleurs être appréhendé dans sa dynamique : les caractéris- 
tiques du client et de la situation peuvent introduire de la nuance, que viendrait par exemple éclairer un travail ethnographique lors des consultations. Les chirurgiens sont en effet amenés à interagir en situation avec celles et ceux qui sont justement catégorisés, rappelant que les médecins n'utilisent pas un unique modèle de prise de décision, mais disposent au contraire d'une pluralité de registres de jugements et d'actions (Baszanger, 1995). Ainsi, la désignation pathologique comme l'invocation du naturel ne sont pas systématiquement sollicitées pour refuser ; et pour cause, des hommes sont opérés. Il faut ainsi distinguer ce qui s'apparente aux discours et aux pratiques.

Mais, selon nous, ce sont surtout les transformations politiques du monde médical qui troublent l'homogénéité de ce monde, en autorisant un changement dans le rapport qu'entretiennent clients et médecins. Certes, la place occupée dans la hiérarchie professionnelle, les stratégies de gestion de carrière, ainsi que les représentations influent sur les positions de chacun de ces professionnels. Mais la modernité thérapeutique influence vraisemblablement les nouvelles générations de praticiens, laissant davantage de latitude aux clients pour investir le choix de leurs opérations. Ainsi, les chirurgiens refusant de manière inconditionnelle les pénoplasties, plus âgés, n'ont jamais cédé à ces demandes, alors que les tenants de l'accord a priori sont plus jeunes. Pour autant, nous ne suggérons pas qu'un modèle remplace l'autre. Et pour cause : il n'y a pas d'homogénéisation absolue des discours en fonction des catégories d'âge, mais plutôt des tendances qui se dessinent. Il est à ce titre important de noter que la culture de ce groupe, exprimée entre autres par ces discours, joue un rôle de tampon dans l'évolution de ces postures. Elle offre les honneurs à ceux qui s'y sont conformés (par l'attribution de postes prestigieux au sein du syndicat ou de la société savante) ou elle pénalise ceux qui s'en écartent, pour lesquels les possibilités d'expression en congrès ou dans la revue scientifique sont limitées dès lors qu'il s'agit de présenter des travaux sur des opérations délégitimées.

Liens d'intérêts : aucun. 


\section{RÉFÉRENCES BIBLIOGRAPHIQUES}

Aubé S., Thoër C., 2010, La construction des savoirs relatifs aux médicaments sur Internet : étude exploratoire d'un forum sur les produits amaigrissants utilisés sans supervision médicale, In : Renaud L., ed., Les médias et la santé. De l'émergence à l'appropriation des normes sociales, Québec, Presses de l'Université du Québec, 239-266.

Austin J.L., 1970, Quand dire, c'est faire, Paris, Le Seuil.

Baszanger I., 1995, Douleur et médecine. La fin d'un oubli, Paris, Le Seuil.

Barbot J., 2008, Soigner en situation de risque judiciaire. Refus de transfusion et responsabilité médicale, Revue Française de Science Politique, 58, 6, 9851014.

Bercot R., Mathieu- Fritz A., 2006, Les chirurgiens. Eléments pour une analyse sociologique d'une crise de recrutement professionnel, Contrat de recherche entre l'AP-HP et le LATTS et GTM, nº 05CTR024.

Bordo S., 1989, The body and the reproduction of femininity: a feminist appropriation of Foucault, In : Jaggar A., Bordo S., eds, Gender/body/ knowledge, New Brunswick, Rutgers University Press, 13-33.

Bourdieu P., 1982, Ce que parler veut dire. L'économie des échanges linguistiques, Paris, Fayard.

Broussard V., Loriol M., Caroly S., 2006, Catégorisation des usagers et rhétorique professionnelle. Le cas des policiers sur la voie publique, Sociologie du Travail, 48, 2, 209-226.

Cassell J., 1998, The woman in the surgeon's body, Cambridge MA, Harvard University Press.

Clement S., Membrado M., 2001, Des alcooliques pas comme les autres ? La construction d'une catégorie sexuée, In : Aïach P., Cebe D., Cresson G., Philippe C., eds, Femmes et hommes dans le champ de la santé. Approches sociologiques, Rennes, Editions ENSP, 51-74.

Cresson G., 1998, Le travail domestique de santé, Paris, L'Harmattan.

Damien R., Tripier P., 1994 Rhétoriques professionnelles, In : Lucas Y., Dubar C., eds, Genèse et dynamique des groupes professionnels, Lille, Presse Universitaire de Lille, 245-247.

Dodier N., 2003, Leçons politiques de l'épidémie du sida, Paris, Editions de l'EHESS.

Fainzang S., 1999, La construction culturelle de la norme et de la pathologie, Confrontation entre Science et Vivant, 1, 9-12.

Fox Keller E., 1992, Essays on langage, gender and science, New York, Routledge 
Ghigi R., 2007, Di virtu' necessita. La chirurgica plastitica in Italia, Thèse pour le doctorat de sociologie, Università degli Studio di Trento.

Guyard L., 2002, Consultation gynécologique et gestion de l'intime, Champ Psychosomatique, 27, 81-92.

Hagège J. C., 1988, La rhinoplastie avant 18 ans et après 40 ans, Annales de Chirurgie Plastique Esthétique, 33, 3, 295-297.

Hugues E., 1996, Le regard sociologique. Essais choisis, Paris, Editions de l'Ecole des Hautes Etudes en Sciences Sociales.

Jacobson N., 2000, Cleavage. Technology, controversy, and the ironies of the manmade breast, New Brunswick, Rutgers University Press.

Jaisson M., 2002, La mort aurait elle mauvais genre ? La structure des spécialités médicales à l'épreuve de la morphologie sociale, Actes de la Recherche en Sciences Sociales, 143, 3, 144-152.

Karpik L., 1995, Les avocats. Entre l'État, le public et le marché, Paris, Gallimard.

Katz P., 1999, The Scalpel's edge. The culture of surgeons, Boston, Allyn and Bacon.

Kaufmann J.C., 1996, L'entretien compréhensif, Paris, Armand Colin.

Knipper P., Jauffret J.L., 2003, Instantané esthétique : le questionnaire sur les interventions et leurs complications, Annales de Chirurgie Plastique Reconstructrice et Esthétique, 48, 2, 299-306.

Lacqueur T., 1992, La fabrique du sexe. Essai sur le corps et le genre en Occident, Paris, Gallimard.

Le Breton D., 1992, Des visages. Essai d'anthropologie, Paris, Métailié.

Le Breton-Lerouvillois G., 2007, L'atlas de la démographie médicale en France, Situation au 1 ${ }^{\mathrm{er}}$ janvier 2007, Ordre National des Médecins, Etude, 40.

Levignac J., 1988, Construction de la face esthétique et perception, Dysrythmies morphologiques et eurythmie, Annales de Chirurgie Plastique Esthétique, 33, 1, 66-78.

Loriol M., 2003, Faire exister une maladie controversée : les associations de malades du syndrome de fatigue chronique et Internet, Sciences Sociales et Santé, 21, 4, 5-33.

Méadel C., Akrich M., 2010, Internet, tiers nébuleux de la relation patientmédecin, Les Tribunes de la Santé, 29, 4, 41-48.

Morgan K., 1998, Women and the knife: cosmetic surgery and the colonization of women's bodies, In : Weitz R., ed., The politics of women's bodies, Oxford, Oxford University Press, 164-183. 
Oudshoorn N., 1994, Beyond the natural body: an archaeology of sex hormones, New York, Routledge.

Pitts-Taylor V., 2007, Surgery junkies, wellness and pathology in cosmetic culture, New Brunswick, Rutgers University Press.

Sarradon-Eck A., Pellegrini I., 2012, Le traitement adjuvant du cancer du sein par tamoxifène. Entre risques et bénéfices thérapeutiques, Sciences Sociales et Santé, 30, 1, 47-71.

Strauss A., 1992, La trame de la négociation. Sociologie qualitative et interactionnisme, Paris, L'Harmattan.

Tamagne F., 2002, Genre et homosexualité, de l'influence des stéréotypes homophobes sur les représentations de l'homosexualité, Vingtième Siècle. Revue d'Histoire, 75, 61-73.

Vilain R., 1987, Jeux de mains, Paris, Arthaud

Weller J.M., 1994, Le mensonge d'Ernest Cigare. Problèmes épistémologiques et méthodologiques à propos de l'identité, Sociologie du Travail, 36, $1,25-42$.

Zolesio E., 2012, Chirurgiens au féminin ? Des femmes dans un métier d'hommes, Rennes, Presses Universitaires de Rennes. 